\title{
Neutron star equations of state with optical potential constraint*
}

\author{
S. Antic ${ }^{1,2}$ and S. Typel ${ }^{1}$ \\ ${ }^{1}$ GSI, Darmstadt, Germany; ${ }^{2} \mathrm{TU}$, Darmstadt, Germany
}

Many properties of nuclei and nuclear matter can be well described with modern phenomenological energy density functionals (EDF). A particular strong constraint represents the observation of two pulsars of approx. two solar masses [1]. It requires a sufficiently stiff equation of state $(\mathrm{EoS})$ at densities well above the nuclear saturation density. Since nucleons of large momenta interact with the medium under these conditions, the momentum dependence of the effective interaction is probed. Experimental information on this dependence is given by the optical potential $U_{\text {opt }}$ that can be extracted from elastic proton scattering on nuclei in Dirac phenomenology [2].

Standard covariant EDF predict a much too strong energy dependence of $U_{\text {opt }}$. Thus, extensions with derivative nucleon-meson couplings have been developed that respect the optical potential constraint. A linear energy dependence of the nucleon self-energies in early models [3] was recently extended to more general functional forms [4]. In the present work, the latter approach with nonlinear derivative (NLD) couplings was combined with a density dependence (DD) in order to obtain a very flexible EDF. This DD-NLD model provides the equation of state at high densites for all neutron-proton asymmetries. The model can be applied to the description of cold charge-neutral matter in $\beta$ equilibirum by adding the contributions of electrons. The mass-radius relation of neutron stars is finally obtained by solving the Tolman-Oppenheimer-Volkoff equations [5] with this stellar matter EoS.

In figure 1 the mass-radius relation of neutron stars is depicted for five models. The first (D1) is a conventional covariant EDF parametrization with density-dependent couplings close to a model that successfully describes properties of finite nuclei [6]. It reproduces well-determined nuclear matter parameters at saturation and gives a maximum neutron-star mass well above the observed masses. However, it does not fulfill the optical potential constraint. The other two models, with identical nuclear matter parameters to model D1, are parametrizations of the full DDNLD model that predict an energy dependence of the optical potential. In model D2, the nucleon self-energies show a Lorentzian form of the energy dependence and in model D3 an exponential dependence is used. The parameter $\Lambda$ regulated the strength of the energy dependence. Here, representative values are chosen that give optical potentials consistent with Dirac phenomenology at high nucleon energies, for details see reference [7]. The resulting EoS of

* Work supported by the Helmholtz Association through the Nuclear Astrophysics Virtual Institute (VH-VI-417) and by the Helmholtz Graduate School for Hadron and Ion Research (HGS-HIRe). these parametrizations are considerably softer than that of model D1 and, consequently, much lower maxium neutron star masses are predicted barely reaching masses of ordinary neutron stars.

In the future, the DD-NLD model will be applied to the description of finite nuclei. It remains to be seen whether successful parametrizations for nuclear structure calculations can consistently satisfy the optical potential and maximum mass constraints.

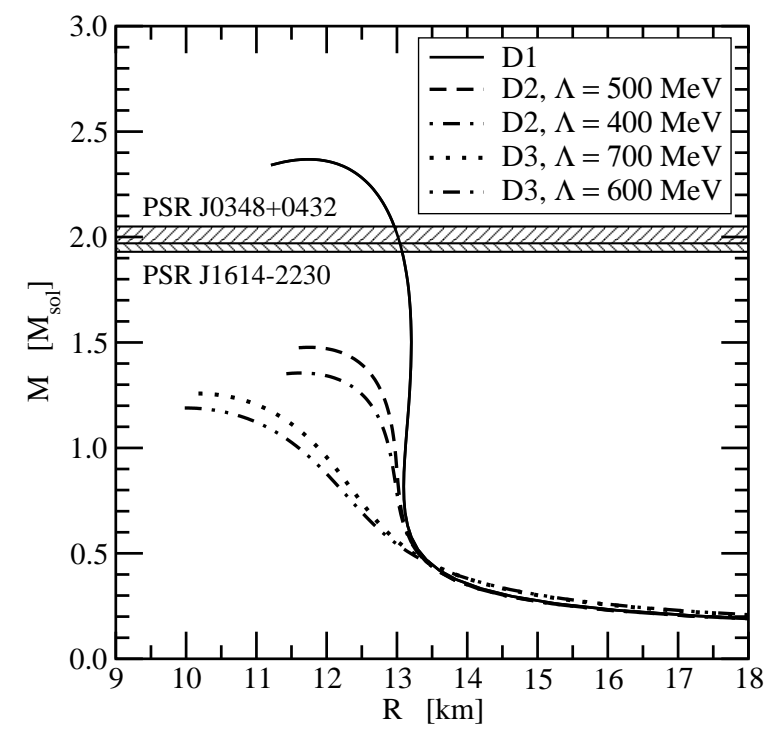

Figure 1: Mass-radius relation of neutron stars for different versions of the DD-NLD model. The shaded bands represent astrophysical mass measurements of two pulsars [1].

\section{References}

[1] P. Demorest et al., Nature 467 (2010) 1081; J. Antoniadis et al., Science 340 (2013) 6131. P. Demorest et al., Nature 467 (2010) 1081.

[2] S. Hama et al., Phys. Rev. C 41 (1990) 2737; E.D. Cooper et al., Phys. Rev. C 47 (1993) 297.

[3] S. Typel et al., Phys. Rev. C 67 (2003) 034002; Phys. Rev. C 71 (2005) 064301.

[4] T. Gaitanos et al., Nucl. Phys. A 828 (2009) 9; Nucl. Phys. A 878 (2012) 49; Nucl. Phys. A 899 (2013) 133.

[5] R.C. Tolman, Phys. Rev. 55 (1939) 364; J.R. Oppenheimer and G.M. Volkoff, Phys. Rev. 55 (1939) 374.

[6] S. Typel et al., Phys. Rev. C 81 (2010) 015803.

[7] S. Antic and S. Typel, arXiv:1501.07393[nucl-th]. 